\title{
Pursuit and Redemption: A Myth-Archetypal Interpretation of the Ferryman
}

\author{
Meng Qiao ${ }^{1, \mathrm{a}}$, Tan Jing ${ }^{2, \mathrm{~b}^{*}}$ \\ ${ }^{1}$ International College, GuangZhou College of Commerce, GuangZhou, China; St. Paul University Philippines, \\ Tuguegarao City, Philippines \\ ${ }^{2}$ Department of Foerign Language, GuangZhou College of Commerce, GuangZhou, China \\ a86779513@qq.com \\ ${ }^{b^{*}}$ mengqiao866@163.com
}

\begin{abstract}
The Ferryman is a novel of spiritual salvation that integrates myth and reality. It centers on Dylan's journey of soul searching and returning, and portrays how ordinary little people get out of the spiritual dilemma and reshape themselves through the pursuit of spiritual home and the test of soul. The author continues the creation mode of pursuing motif and salvation prototype in ancient Western mythology, and adopts innovative displacement in the new background. It reflects the author's positive thinking about the fate of human beings in the modern context, enriches the form and connotation of myth paradigm and has profound significance on different cultures and times.
\end{abstract}

Keywords: Ferryman, archetypal criticism, pursuit, salvation

\section{追寻与救赎: 《摆渡人》的神话-原型解读}

$$
\text { 蒙巧 } 1, a \text { 谭静 } 2, b^{*}
$$

\footnotetext{
1 广州商学院国际学院, 广州, 中国; 菲律宾圣保罗大学, 土格加劳市, 菲律宾

2 广州商学院外国语学院, 广州, 中国

a86779513@qq.com

b*mengqiao866@163.com
}

\section{摘要}

《摆渡人》是一部融神话与现实为一体的心灵救赎小说，围绕女主人公迪伦出走与回归的灵魂穿越之旅，塑造 了普通小人物通过对精神家园的追寻和灵魂的洗礼，走出精神困境，重塑自我。作者延续了古代西方神话中的 追寻母题与救赎原型的创作模式，在新的时代背景中对人物追寻模式及叙事程式进行大胆的移位，彰显了作者 对现代语境下人类命运的积极思考, 丰富了神话范式的形式与内涵, 具有跨文化与跨时空的深远意义。

关键词: 《摆渡人》; 追寻; 救赎; 置换

\section{1.前言}

“原型”(archetype)一词由希腊文 arche 与 type 构 成,指事物的模子或最初形式, 也引申为典型, 或文学、 艺术、神话、梦以及仪式中反复出现的行为范式、意 象或主题 (Abrams 1999: 12)。瑞士心理学家卡尔·荣 格（Carl Gustav Jung, 1875-1961）认为，原型或原始 意象，是一种“在历史进程中不断发生并且显现于创 造性幻想”的形象一一魔鬼、一个人或一个过程, 蕴含 着“人类精神和命运”, 有着在我们祖先历史中“重复
了无数次的欢乐和悲哀的”的经验, 艺术家的创作过 程，就是“从无意识中激活原型”并“加工造型精心制 作”，使同时代的我们“有可能找到一套道路以返回生 命的最深的源泉”（2014:84-86）。加拿大原型批评的 集大成者诺思洛普·弗莱(Northrop Frye,1912-1991)认 为，原型是“一种典型、反复出现的意象”, 它“把一首 诗同其他诗联系起来”从而有助于统一人类文学经验 的象征（2011a: 155）；神话世界是一个“充满情节虚 构和主题构想的非实在的”文学结构, 是一个“整体的 隐喻世界”, 要使现实主义作品中所蕴含的神话结构 
使读者“信以为真”, 就要通过置换变型 (displacement) 技巧（2011b: 171）。简言之，神话是最基本的原型模 式，而其他模式只不过是神话的移位或变异。读者 若要探索各类文学现象与古代神话、原型意象之间的 密切联系, 需要“从神话着手从宏观上研究文学艺术 自身的内在类似性, 即其程式, 结构模式和原则, 并 从整体上探寻文学类型的共性和演变规律” (张中载 2006: 829）。在找到作品的原型之后, 还须联系特殊 的时代背特征景或特定的文化语境, 才能深入分析置 换的神话与隐喻, 从而真正把握作品的思想内涵。

《摆渡人》 ${ }^{(1)}$ (Ferryman, 2013) 是英国文坛当 代著名女作家克莱儿·麦克福尔 (Claire McFall, 1983-,以下简称“麦克福尔”）创作的一部心灵治愈 经典之作, 自 2013 年出版以来, 获得五项世界文 学大奖，版权销售 33 个国家，曾居中国畅销书排 行榜, 读者众多, 但对其进行深入研究的学者不多, 主要从荒原意象、主题的多样性（爱情、亲情、死 亡、成长等主题）、叙事结构等方面进行探讨与评 论。例如在叙事模式上, 国内有学者从物理空间、 心理空间和社会空间视角阐释了《摆渡人》的双重 叙事结构, 认为小说中迪伦在荒原中的探索是空间 “追寻和救赎”的隐喻, “折射人类困境及自我救赎之 举”（池铭琛 2018: 41）。

总体而言, 把《摆渡人》与神话原型批评系统联 系起来研究的并不多, 大多都是在论述其他主题中零 星地提及。麦克福尔多次谈及其创作灵感主要来源 于她对于人死之后会发生什么的好奇和对希腊神话 中冥府渡船人的故事。受西方希腊神话的影响, 或许 使得她的作品自觉或不自觉地直于古代神话原型模 式。那么, 麦克福尔是如何在《摆渡人》中借鉴与延 续古代西方神话中的原型模式呢? 本文试图厘清该 小说的人物原型模式和叙事原型模式, 探讨作家在现 代社会语境中如何对人物追寻模式及叙事程式进行 大胆的置换及关于置换的潜在哲思, 从而挖掘小说深 层的主题和内涵。

\section{2.人物原型模式的移位：追寻的神话}

记录人类生命体验的追寻之旅是西方文学作品 中常见的母题之一。自亚当夏娃偷食禁果被逐出伊甸 园, 漂泊流浪与寻找归途似乎成了人类祖先与生俱来 的宿命。在文学历史从古到今的发展中, 人类的追 寻经验经历了诸多变化, 从追寻具体的人或物品, 演 变到对灵魂归宿、人生真谛和生命意义的探求等, 典 型的代表作有荷马史诗《奥德赛》(Odyssey)、歌德的 《浮土德》(Faust), 班扬的《天路历程》(The Pilgrim's Progress), 詹姆斯·乔伊斯的《尤利西斯》(Ulysses), 卡夫卡的《城堡》 (The Castle), 丹·布朗的《达·芬奇 密码》(The Da Vinci Code) 等。

《摆渡人》讲述了成长于单亲家庭的 15 岁女孩 迪伦离家出走, 乘火车去探寻十年未谋面的父亲, 因 突发交通事故意外死亡后，在摆渡人崔斯坦的保护与
引领下，其灵魂穿越荒原到达“家”，后因对崔斯坦的 滕恋而又重返荒原、回到人间的奇幻之旅。笔者认为, 麦克福尔在刻画迪伦这一形象时, 是对古希腊英雄奥 德修斯离家与返乡追寻故事模式的延续与置换变形, 巧妙结合作家对希腊神话的领悟与对现代生活的哲 思, 探索现代语境下人们对生死、命运等思考与抉择 的精神历程。“共性是移位产生的基础” (王香玲 2016: 74）。那么, 《摆渡人》中原型人物奥德修斯 与变形人物迪伦之间存在哪些本质上的联系与区别 呢?

一方面, 原型人物奥德修斯与变形人物迪伦的出 走与回归模式如出一辙, 二者都经历了 “离家-战争 (磨难)-回家”的心路历程。奥德修斯是伊萨卡岛的统 治者, 应阿卡门农的请求, 离开刚出生的孩子, 参与 对特洛伊的战争, 巧用木马计攻破顽抗十年的特洛伊 城, 最后带领部下开始了漫长的回乡之旅, 在海上漂 泊十年, 先后降伏魔女喀耳刻, 克服塞壬仙女美妙歌 声的诱惑, 穿过巨人波吕斐摩斯的居地, 拒绝神女卡 吕普索的永生许诺, 坚定归乡, 最后同儿子一起杀死 逼迫他妻子的求婚者，周“家”团圆。这里的“家”可以 说是一种隐喻, 可以指“国家”“家园”“家人”，甚至是 一种坚定的“信念”，永恒的爱，或理想的精神家园。 几千年后，现代女孩迪伦踏上了同样的征程。在现实 世界中, 迪伦和母亲因缺乏交流而逐渐疏远, 在学校 里没有朋友而倍感孤独寂寞, 于是决定离家出走, 去 寻找久未谋面的父亲, 不幸在隧道遭遇火车事故, 在 摆渡人的引领下, 在荒原中穿越山谷, 智斗恶魔, 险 渡黑湖, 到达灵魂之“家”, 却因对崔斯坦的留恋, 重 返荒原, 最终引渡崔斯坦一起返回现实世界。显而易 见, 出走与回归的“追寻”经验是原型人物与变型人物 之间的本质联系。通过文本细读, 可以在两位主人公 的经历中找到很多熟悉的故事元素, 如主人公经历惊 险、冲突、痛苦、挣扎甚至逼近死亡，但二者都展现 了坚强的意志与非凡的勇气, 最终成功实现身心的蜕 变。

另一方面, 变形人物与原型人物在时代背景、人 物身份、性格特征等方面却又截然不同。

变形人物是在原型人物基础上创造出来的具有 时代特色的人物, 同样是“追寻”英雄, 两者在人物表 现形式上存在明显的差异。奥德修斯是希腊神话中 本真大英雄, 他是伊萨卡岛的国王, 是神与人结合的 后裔, “身躯伟岸”“面容神色奕奕, 双侠丰满, 头发稠 密”, 看起来像“神祇”（弗拉克斯曼 2018: 540）。冊 庸置疑, 奥德修斯拥有英雄人物所特有的优秀品质, 对国家、部落的责任感、骁勇善战、拥有无上的权威, 带领部下, 打败对手, 屡建奇功, 十年漂泊, 矢志不 渝, 重返故土。然而, 当时光回溯到现代社会中, 故 事的主角因时而异，被降格置换成了吉斯夏尔中学女 学生迪伦。“在英雄已逝的舞台上, 反英雄即在陌生孤 寂的世界里显得渺小无能的小人物正扮演着主 角”(弗莱，1998b: 235)。与奥德斯修不同，生活在现 代社会的小人物迪伦普通得像尘埃, 缺乏远大的目标, 
没有崇高的壮举，“做事一点计划都没有”; 性格 “内 向差涩”, “不是特别聪明”, 且有些优柔寡断, 在学校 里“一直都在避免成为别人关注的焦点”; 对现实不满 却又缺乏足够的勇气去反抗, “知道反抗是徒劳的”(48)。正是这样对现实无能为力的小人物, 却要担当英 雄的使命, 成为“现代版”追寻精神家园的主角, 开启 其惊心动魄的灵魂之旅。

同时, 原型人物与变形人物的的追寻之旅都得到 了外力帮助, 尽管帮助者大不相同。作为古代英雄的 奥德修斯带领部下历经艰险而追寻家园，途中自然少 不了同伴间的全力以赴, 但天神的帮助在关键时刻起 了重要作用, 包括风神埃俄罗斯赠予的能关各式各样 风的风袋助其还乡; 宙斯赐予他可防止变成动物的草 药, 才得以从女神卡吕普索的岛上离开; 雅典娜用金 色神杖助他化身乞正迷惑众人最终夺回家园等。奥德 修斯充满智慧且行动果敢, 但如若没有伙伴的帮忙, 众神的或明或暗的眷顾指点, 他是很难独自一人逃脱 所面临的巨大威胁。

相比之下，现代语境下的迪伦的“追寻”之旅，从 一开始就注定是势单力薄的。没有家人的支持, 母亲 “本就对迪伦在周末去见她父亲十分不满” (4); 没有 伙伴的倾诉, “自从凯蒂走了以后, 这所学校所有人给 人的感觉就像跟迪伦不住在同一个星球一样, 更别说 是同一物种了”（7）; 即使在去见父亲的途中，也与 周围的人格格不入, 与他人的社交性聊天对她都是一 种折磨, “她急不可耐第想从眼前的社交游戏中解脱 出来”(12), 火车里邻座女人对她也“高声叹了口气, 不满之情溢于言表” (13)。唯一帮助迪伦的, 只是火 车发生事故之后, 等待摆渡她的男孩崔斯坦, 但他只 是一个身不由已的摆渡人, “在他之上还有更高的自 然法则, 还有不可撼动的命运......他无法掌控自己的 命运, 他甚至无法控制自己的双脚”（167）。欲想穿 越荒原, 实现灵魂的成长, 迪伦更多的是需要自己的 内省与坚持, 甚至反转成为摆渡人崔斯坦的摆渡人, 改变其对命运与生命意义的认知, “由受助者转为帮 助者, 对崔斯坦实施帮助”（池铭琛 2018: 43）。

纵观原型人物与变形人物的追寻经验, 爱、责任、 意志、勇气与信念都是两者成功的重要因素, 但英雄 人物原型在追寻中更多借助不可抗的神力因素, 而现 代社会背景下的变型人物, 经历更多的是心灵的成长 与自我救赎。

\section{3.叙述原型模式的置换: 救赎与重生的神话}

诺思洛普·弗莱用原型批评的方法解析基督教经 典《圣经》, 在其著作《伟大的代码一圣经与文 学》中归纳提出 $U$ 型叙事结构, 认为整部《圣经》 的故事发展与叙事脉络, 从开篇《创世纪》讲述人 类失去生命和水, 到《启示录》末尾而又复得, 遵 循了下降（落难）一悔悟（死亡）-重升（复活/救赎 /重生）到差不多相当于上一次下降时的高度的 U 型 结构的叙述模式。创世纪之初, 人类始祖亚当和夏
娃快乐地生活在伊甸园中, 因禁不住蛇的诱惑偷吃 禁果而被逐出伊甸园, 经历了逃亡、灾祸与苦难, 等到耶稣受难替人类赎罪后, 得以重新获得曾经失 去的美好家园。这种近似 $U$ 型弧线的叙事模式被弗 莱称之为圣经的叙事结构单位, 具有稳定性, 反复 出现在喜剧类文学作品中。在此叙事模式下, 一系 列的不幸遭遇会使情节发展到危难的最底端, 而后 某种有利的线索又使结局得以扭转。可以说, $\mathrm{U}$ 型 结构的左边下降部分代表主体遭受苦难, U 型结构 的底端往往体现于主体象征意义上的死亡, 而 $U$ 型 结构右边的上升部分代表主体复活或救赎, 是重生 母题神话的典型描述。

在《摆渡人》中，麦克福尔的叙事笔墨围绕着主 人公迪伦 “出走寻找父亲-灵魂荒原之行-重返现世家 园”三次经历层层展开, 呈现出近似耶稣复活的完整 的 U 型路线。显而易见, 耶稣复活宏大庄重的经典 叙事经由现代社会普通小人物迪伦的灵魂之旅得以 重演, 正是在这样跌宕起伏、复杂多变的叙事中, 小 说追寻、救赎与重生的主题与内涵得以不断深化。

小说第一部分以迪伦离家出走寻找父亲作为 $\mathrm{U}$ 型结构故事的起点。寻找父亲之路, 字面上是去与缺 席的父亲会面, 其实质是对现实困窘生活的抛弃和对 自我主体性认知的隐喻, 折射出迪伦自我觉醒之萌动, “是寻爱之旅, 更是自我救赎的旅程” (池铭琛, 2018: 41）。寻父途中迪伦在火车隧道遭遇交通事故死亡, 这是追寻过程的重要转折点, 也是 $U$ 型结构左边下降 部分, 作者叙述的基调是灰暗的, 死亡、黑暗、恐惧、 疼痛等字眼不断撞击着读者的视野:

$$
\text { “一片死寂。 }
$$

应该有的尖叫声啊、哭喊声啊, 总该有点动静 吧。迪伦想。

\section{但是周围只有死寂。}

渌黑一片, 黑暗如一条厚厚的毯子一样笼罩着 她。一瞬间恐惧袭来, 她以为自己失明了。她狂躁 第在脸前挥舞着手, 什么也看不见, 她设法用手唯 了翟眼睛, 刺痛产生的震惊让她思索了片刻。”

(18)

在小说第二部分, 迪伦的灵魂荒原之行经历着 $\mathrm{U}$ 型结构的左边下降部分, 代表主体将遭受磨难, 经历 重重考验。途中, 他们要徒步穿越阴暗的山谷, 蹚过 沼泽与烂泥, 渡过粘稠的黑湖, 随时有被魔鬼吞噬的 危险, 追寻之旅异常艰险。作者叙事的基调是恐惧的, 同时也夹杂着沁入人心的暖意。荒原的经历与洗礼让 迪伦不断得到成长, 和崔斯坦互相换扶一起积极对抗 恶魔, 体验到人与人之间的爱与温暖, 这给迪伦极大 的安慰与信心, 变得果敢和坚强。特别是渡黑湖时, 迪伦曾一度掉进湖里溺水, “当她逐渐陷入昏迷的时 候, 脸上还带着绝望呐喊的神情”（144），幸而崔斯 坦及时施救, 她 “终于带着满心的恐惧感醒来了”

(146)。迪伦掉进黑湖获救是整个叙事的重要转折 
点, 与基督教教义里洗礼相呼应, 隐喻主体的死亡和 重生, 是故事的高潮部分, 也是 $U$ 型叙事结构的底 端。

如果说迪伦因掉进黑湖洗礼而获得新生, 小说的 第三部分则是迪伦新生后的救赎--重返现世家园。当 迪伦经历千辛万难后穿越荒原分界线, 到达所有灵魂 都梦摪以求的“家”时, 却意识到“她不属于这里”, “不 应该呆在这儿”（182），因为没有崔斯坦陪伴的“家” 对她而言还是“形单影只、落落寡欢”的荒原 (184)。 重返荒原同时也意味着没有摆渡人的保护而独自面 对千难万险, 但是追寻爱的勇气与信念不断鼓励着女 主人公, 崔斯坦的“面庞在她脑中浮现, 无比清晰, 这 让她又有了继续搏斗的力量”（211）。作者叙事的基 调是志忈的, 同时也充满了希望, 这是 $\mathrm{U}$ 型结构叙事 的右边的上升部分, 代表着主体的救赎。这里的救赎 有两层含义。一是主人公迪伦的自我救赎, 从以前的 缺少关爱、差澀内向、逃离现实的弱小女孩, 成长为 内心勇敢坚强、为爱甘于奉献、对现实世界重塑信心 的个体。二是主人公对他者的救赎, 包括重返荒原中 冒着生命危险救出一个小女孩免遭恶魔之手, 也包括 对崔斯坦的救赎, 鼓励他摆脱荒原的禁锢, 打破荒原 上的规则, 重返人世间, “他们穿越荒原, 越走越 远 ......迪伦感觉到自己正在分离挣扎重获新生” (261)。

迪伦和崔斯坦二次成功穿越荒原重返现世家园, 与小说的开头相呼应, 让故事回升到与起点差不多的 高度, 形成一个完整的 U 型结构的右上边。作者在 此部分的叙事基调是欢快的。在小说的最后, 迪伦与 崔斯坦因完成各自的救赎圆满相遇于现世家园。“海。 原来你在这里。”“我在这里。”(274) 为迪伦充满惊 险、爱与信念的追寻之旅画上了圆满的句号, 体现了 主人公追寻经验后的心灵成长与自我蜕变, 是一种美 好情感的升华, 也体现了作者对人类社会抱有的爱和 希望。

尽管耶稣复活与迪伦的精神之旅都呈现出极其 相近的 U 型发展结构, 但在这一表象下仍可以清晰窥 测到二者的区别。第一个区别是重生的形式。耶稣的 复活是实体的死亡与重生, 迪伦虽然在车祸中经历死 亡而最终又成功返回现实界, 然其“复活”更多地指向 心灵的顿悟、自省与升华。第二个区别隐没于形式之 外, 即重生的动力与源泉。做为上帝之子的耶稣, 可 以凭借上帝的力量得以复活, 其动力源泉来自对上帝 的信仰与对人类罪恶的救赎。那么, 是什么核心力量 促使迪伦直面生死、穿越荒原从而实现灵魂蜕变呢? 诚然，摆渡人崔斯坦、已渡灵魂到“家”的乔纳斯和伊 扎特在迪伦灵魂的的穿越中起来不可否认的作用, 但 这都属于外力的辅助作用。真正促使她内心一变的核 心力量则是源于西方传统文化中所称颂的“希望”，即 对于人类命运所持有的永不泯灭的笃信。恰如潘多拉 打开魔盒, 尽管各种邪恶与苦难肆虐, 但在盒子盖上 的一刻希望即已封存, 预示着人类将在痛苦与希望中 不断前行。小说中, 面对荒原的恶魔与阴影, 迪伦曾
不免恐惧与沮丧, 但她始终坚信自己要去的地方 “肯 定是个好地方”（80），说服摆渡人崔斯坦一起重返现 实世界，笃定自己的选择是“以前从没有人尝试过的 事情“（256），返回列车事故现场时面对摆渡人的质 疑与犹豫, 始终不言放弃, 因为她“心里有希望”“还有 爱”（265）。在麦克福尔充满想象力的笔触下, 在这 场向死而生的生命轮回中, 对人类美好的希冀给迪伦 的灵魂之旅注入了巨大的勇气。耶稣因对上帝的信 仰和为人类赎罪而复活, 迪伦凭借对人类命运的希望 和信念而获得新生。

\section{4.神话置换的哲思}

麦克福尔大胆将古代英雄奥德修斯的追寻母题 置换到现代社会的吉斯夏尔中学, 使之降格成为一个 现代社会普通的女生对父亲、爱与希望的追寻, 并为 主人公的追寻之旅铺设了类似耶稣复活的模式, 庄重 肃穆高大威猛的原型人物与现实社会普通人物的无 力形成巨大的反差, 增强了小说的叙事效果与张力。 随着现代社会理性的崛起, 反映人类祖先愿望幻想的 神话逐渐消亡, “原有的文学不断随着社会发展的步 履而“置换变形”为新的文学” (叶舒宪 2018：72）。 作家对神话的移位是为了使文学本身适应自己的时 代, 无论自觉与否, 往往带有时代的烙印, 难免受到 作家个体价值取向的投射。麦克福尔对古代西方神话 中的追寻母题与救赎原型的创作模式的延续与移位, 折射了作家对现代语境下人类生存状况的关照与思 考。

显然, 作家笔下的“现代版”追寻英雄迪伦, 聚焦 了 21 世纪社会时代特征, 是对神话原型人物模式的 “创造性叛逆”。“创造性叛逆”一词由法国文学社会学 家埃斯卡皮（Robert Escarpit，1918-2000）提出，原 本主要指在文学翻译中, 译者为达到某一主观愿望而 对原作的增减、修改甚至杜撰, 背离了原作的初衷, 但同时又赋予了原作新的生命。笔者认为, 新的文学 作品同样可以对先有文学进行模仿、加工和再创造, 是作家对原有文学模式的“创造性叛逆”。麦克福尔 笔下的迪伦，在时代背景、人物身份、性格特征等表 现形式与原型人物截然不同，没有宏大的叙事，不是 高贵的出身, 缺乏果敢的性格, 背离了传统英雄人物 的形象，但其“出走-追寻-回归”这一人类所共有的经 验模式, 在英雄已逝的现代社会舞台上继续上演, 不 仅延长了原型人物模式的生命力, 同时在新的历史背 景下赋予了新作品崭新的面貌和意义。“现代版”追 寻英雄迪伦, 是作家对当代社会年轻人的描摹。2 2 世 纪的典型特征是科技与网络通讯的高速发展, 是所谓 的“Z 世代”（张濒武 2021），其整体性特征表现为人 类从小就浸润在数字化虚拟网络空间, 追求新技术, 乐于在线互动, 但对深度内容缺少接受度, 抗拒统一 的、逻辑规整的表达。可以说, 网络化、智能化、虚 拟化、速度、变化、危机等成了这一时代代名词。在 此特征之下，人们的生活与手机、平板、电脑等电子 屏幕难以分割, 低头族随处可见, 沉浸在各自虚拟的 
网络世界，人类零碎的时间已被电子信息、游戏、视 频等填满，导致个体之间缺乏深入和坦诚交流，后现 代社会人类生存的精神荒原拔地而起, 个体或群体好 似被抛弃在一片无尽的荒野中，忙碌、茫然与孤独并 存。小说开始, 母亲就责备迪伦沉迷于网络: “昨天晚 上是不是又碰电脑了? ”, 总是 “在 MSN 上胡闹” (34）。的确，相比于传统的交流方式，迪伦更喜欢通过 网络的方式与好友分享最新个人体验, 学校教室对于 她来说显得“满目倦容”,充满着压抑感，而“屋子里坐 了三十个人形机器人, 个个都在说着毫无价值的废话, 就好像正在演一出能改变生活的大戏” (5) ; 人与人 之间关系疏远冷漠, “这所学校里所有人给人的感觉 就像跟迪伦不住在同一个星球一样, 更别说是同一物 种了”（7）。通过迪伦之眼, 作家勾画出了 Z 世代之 下人与社会、人与他人、人与自我之间存在的困境。

另外, 作家笔下追寻英雄的性别置换: 由至高无 上的“男性英雄”变成了现代社会普通学校的普通女 生, 反映了作家对当下女性角色的思考。众所周知, 古希腊神话塑造了大量的丰富多彩的人物形象。然 而, 从盖亚(Gaea)、乌拉诺斯 (Uranos)、克洛诺斯

(Cronus) 到宙斯（Zeus）之间神权的更替，不难发 现早在古希腊时期, 女性已逐渐沦落为男子的附属, 处于社会的底层, 成为被边缘化的弱势群体, 恰如恩 格斯所言:“神话中女神的地位表明, 在更早的时期, 妇女还享有比较自由和比较受尊敬的地位, 但是到了 英雄时代, 我们就看到妇女的地位已经由于男子的统 治和女奴隶的竞争而降低了”(1972:32）。以上论断 从目前图书市场的各类版本的希腊神话故事和英雄 故事传说等书也得到了印证, 这些神话故事选编书籍 的目录大多是以男性英雄人物命名, 且故事基本是围 绕男性主人公的丰功伟绩、探险、战争、荣耀等展开, 女性往往处于配角、祄托的角色。以古斯塔夫.施瓦布 的《希腊神话与传说》 (2018) 为例, 在长达 725 页 的故事文本中, 其目录包括 5 部分 69 个二级标题, 只有 9 个二级标题直接或间接以女性名字命名 ${ }^{2}$, 其 他的全部是男性名字或男性相关的事件命名, 故事也 是围绕男性主人翁而层层铺开。时光荏冓, 当时间之 轴转向 21 世纪的英国, 风靡一时的《哈利·波特》, 销售榜首的《摆渡人》均出自女性作家之笔, 甚至一 度女王和首相 (Theresa Mary May)都是同时代的女性, 都可窥见女性在历史发展中所作的巨大贡献及女性 在社会中地位的不断攀升。无怪乎马云在其《开讲啦》 英国行中断言: 21 世纪是女性的时代 ${ }^{(3)}$ 。在此背景下, 作为职场女性 (中学英语教师) 兼作家的麦克福尔, 对追寻英雄人物性别大胆的置换, 在看似䇔弱的表象 下, 赋予女主人翁极大的信念与勇气, 完成看似不可 能的灵魂救赎, 实现自我蜕变, 不论是出于作家本身 的性别特点, 还是出于人物形象灵感来自所教学生的 思量, 均彰显了作家对现代女性自我成长的思考, 或 者更广泛地说, 在英雄缺失及精神荒原的现代社会, 每一个人，无论男性与女性，都可以化身为神话中的 英雄, 成为自我构建与自我成长的主体。

\section{5.结论}

在小说《摆渡人》中，麦克福尔延续了古代西方 神话中的追寻母题与救赎原型的创作模式, 在新的时 代背景中对人物追寻模式及叙事程式进行大胆的移 位, 通过对古希腊神话中的英雄奥德修斯的置换变形, 借现代社会女主人公迪伦近似 $U$ 型叙事结构的荒原 之旅, 塑造了当代语境下普通小人物对精神家园的孜 孜不倦的追寻, 经历灵魂的成长与洗礼, 走出精神困 境, 重塑自我, 完成蜕变之路, 突出了小说的时代特 征, 深化了小说的追寻、救赎与重生主题。现代作家 在文学创作中采用神话, 实则“托古喻今”, 是对“混乱 的现实加以嘲弄式的把握”，是“一种理解生活的必 要方式” (叶舒宪, 2018:5)。麦克福尔正是借助作品 中人物追寻与 $U$ 型叙事的隐喻, 在现代神话的荒原中 重塑对人类的爱、温暖与希望，直抵人心，书写人类 命运的曙光, 唤起读者对当下社会现实困境的思考和 救赎之路的探寻。在世界科技发展、物质丰盈却信 仰衰微的时代背景下, 作为普通的个体人物, 不论男 性抑或女性，敢于打破社会陈规和自我禁锢，以积极 的态度面对生活, 对自我及他人重塑信心，对人类的 命运永不言弃, 本身就是对人类命运发展的充分肯定 与积极思考。特别在当下新冠肺炎疫情全球肆虐的 特殊背景下, 人类遭遇空前的灾难, 时刻与病毒进行 生死抗争, 人类需要正视灾难和拯救自我, 更需重拾 对生命的敬畏，重塑对社会的责任担当，坚信人类美 好未来的理想, 麦克福尔在《摆渡人》中所展示的积 极的人类命运伦理观可谓切中肯綮。

\section{项目基金}

本文为广东省高校思想政治教育课题“中 外合作办学背景下应用型本科高校课程思政 建设困境与对策研究”（2020GXSZ099）、广州 商学院 2020 年高等教育教学研究和改革项目 “应用型本科高校中外合作办学项目课程思政 教学实践探索”、广州商学院课程思政“四个一” 试点课程《英语阅读》（KCSZSDXM202082） 的阶段性研究成果之一。

\section{注释:}

(1) 克莱尔·麦克福尔.摆渡人(2015) 付强, 译. 南昌: 百花洲文艺出版社。以下该书直接引文只在文中注 明页码，不再一一做注。

(2) 古斯塔夫·施瓦布的《希腊神话与传说》中，以 女性名字命名的 9 个二级标题分别为: “欧罗巴”、 “俄尔普斯和欧律狄刻”、“塞壬仙女”、“珀涅罗珀来 到求婚人面前”、“奥德斯修单独与忒勒玛科斯和珀 涅罗珀在一起”、“惩罚女仆”、“奥德斯修与珀涅罗 珀”、“俄瑞斯忒斯和复仇女神”。 
(3) 马云在《开讲啦》英国行·第 5 讲“商业与女性” 专场的励志演讲稿(总第 162 期), 播出时间: 2015$10-31$ 。

\section{REFERENCES}

[1] Abrams, M. H. (1999) A Glossary of Literary Terms. Foreign Language Teaching and Research Press, Beijing.

[2] Carl Gustav Jung (2014) Psychology andLiterature. Translated by FengChuan, SuKe. Yilin publishing house, Nanjing.

[3] Chi Mingchen (2018) Redemption towards death and life: a review of the double narrative of McFaul's the ferry man. World literature review, (2): 41-45.

[4] Claire McFaul (2015) Ferryman. Translated by Fu Qiang. BaiHuaZhou literature and Art Publishing House, Nanchang.

[5] John Fraxman(2018) Greek Mythology and Legend. Translated by Gao Xiaofu et al. Time literature and Art Press, Changchun.

[6] (Germany) Gustav Schwab (2018) Greek Myths and Legends. Trans. Gao Xiaofu, Guan Huiwen, et al. Time Literature and Art Press, Changchun.

[7] Marx and Engels (1972) Selected works of Marx and Engels (Volume 4). People's Publishing House, Beijing.
[8] Northrop Frye (1998b). Anatomy of Criticism. Trans. Chen Hui et al. Baihua Literature and Art Publishing House,Tianjin.

[9] Northrop Frye(2011b) Archetypal criticism: myth theory. In: Ye Shuxian. Myth archetypal criticism. Shaanxi Normal University Press Co., Ltd., Xi'an. pp.166-196.

[10] Northrop Frye (2011a) As a symbol of archetype. In: Ye Shuxian(ed), Myth archetypal criticism. Shaanxi Normal University Publishing General Office Co., Ltd., Xi'an. pp. 152-165.

[11] Northrop Frye (1998a) The Great Code: Bible and Literature. Trans. Hao Zhenyi et al. Peking University Press, Beijing.

[12] Wang Xiangling(2016) Pursuit and Rebirth: a Myth Archetypal Interpretation of Herzog. Foreign language teaching, 37 (02): 73-77.

[13] Ye Shuxian(2018) Theory and Method of Archetype Criticism. Shaanxi Normal University Publishing General Office, Xi'an.

[14] Zhang Yiwu(2021-01-14 (015)) "Generation Z" becomes an important variable in international relations. Global times.

[15] Zhang Zhongzai(2006) Archetypal criticism. In: Zhao Yifan, Zhang Zhongzai(Eds.), Key Words of Western Literary Theory. foreign language teaching and Research Press, Beijing. pp. 827-836. 